\title{
Endoscopic detection of colonic tuberculosis in an asymptomatic patient
}

There has been a resurgence of intestinal tuberculosis during the last decade following the increase of immigrants to Western countries $[1,2]$. The abdominal symptoms of intestinal tuberculosis are nonspecific, consisting mainly of abdominal pain, weight loss, anorexia, and fever $[2,3]$. The characteristic endoscopic features of colonic tuberculosis include transversely oriented ulcer, nodules, deformed ileocecal valve, stricture, erosions, and aphthous ulcers [1-3]. However, in patients without abdominal symptoms or pulmonary infection, endoscopists face the challenge of distinguishing between intestinal tuberculosis and malignancy or Crohn's disease. We report here a case of colonic tuberculosis found incidentally during a health check-up, and which was diagnosed by typical endoscopic features and histological and microbiological evidence.

A 38-year-old, previously healthy man visited our institution for a scheduled health check-up. His medical history and physical examination were unremarkable. He had a history of travel to China 3 months before coming to our hospital. Chest radiograph showed no active pulmonary lesions. Laboratory data were within reference range except for a triglyceride level of $381 \mathrm{mg} / \mathrm{dL}$ (normal range $50-130 \mathrm{mg} / \mathrm{dL}$ ). Tumor markers including CEA, CA 125 and CA 19-9 were normal. Ziehl-Neelsen stain and culture of sputum were negative for Mycobacterium tuberculosis.

Colonoscopy revealed a transversely oriented ulcer in the cecum, with steep edges and surrounding flared nodules (Figure 1). The terminal ileum appeared normal. Histological examination of the biopsy specimens demonstrated wellformed granulomas with caseous necrosis and Langhan's giant cells (Figure 2). Culture of biopsy specimens revealed positivity for M. tuberculosis, as did the result of polymerase chain reaction for DNA of M. tuberculosis. A diagnosis of colonic tuberculosis was made. The patient receiv-

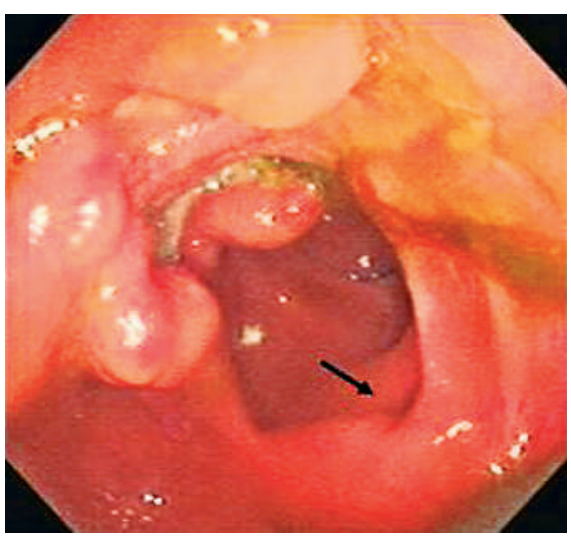

Figure 1 Colonoscopic view showing a transversely oriented ulcer in the cecum with steep edges and flared surrounding nodules. Note its location opposite the ileocecal orifice (arrow).

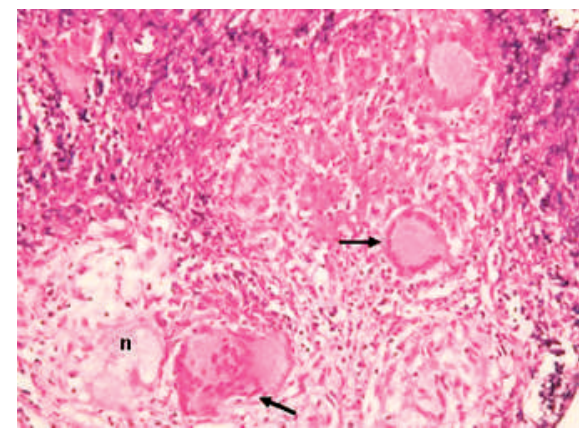

Figure 2 Histological view showing wellformed granulomas with Langhan's giant cells (arrows) and caseous necrosis ( $n$ ), surrounded by a prominent rim of lymphocytes (hematoxylin and eosin; original magnification $\times 100$ ).

ed antitubercular therapy for 9 months with an uneventful clinical course. He remained asymptomatic over the ensuing 2 years of follow-up.

\section{Endoscopy_UCTN_Code_CCL_1AD_2AC}

\section{C. Yang, C. H. Chen, S. L. Yan}

Division of Gastroenterology, Department of Internal Medicine, Show-Chwan Memorial Hospital, Taiwan, Republic of China.

\section{References}

${ }^{1}$ Sato S, Yao K, Yao T et al. Colonoscopy in the diagnosis of intestinal tuberculosis in asymptomatic patients. Gastrointest Endosc 2004; 59: $362-368$

2 Alvares JF, Devarbhavi H, Makhija P et al. Clinical, colonoscopic, and histological profile of colonic tuberculosis in a tertiary hospital. Endoscopy 2005; 37: 351 - 356

${ }^{3}$ Misra SP, Misra V, Dwivedi M, Gupta SC. Colonic tuberculosis: clinical features, endoscopic appearance and management. J Gastroenterol Hepatol 1999; 14: 723 - 729

\section{Corresponding author}

\section{S. L. Yan, MD}

Division of Gastroenterology Department of Internal Medicine Show-Chwan Memorial Hospital No 542, Sec 1, Chung-Shang Rd Changhua 500

Taiwan

Republic of China

Fax: $\quad+886-4-7233190$

Email: yslcsmc@yahoo.com 